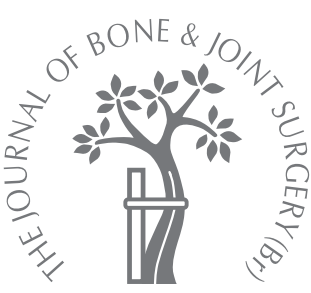

L. B. Solomon,

Y. C. Lee,

S. A. Callary,

M. Beck,

D. W. Howie

From the University

of Adelaide and

Royal Adelaide

Hospital, Adelaide,

Australia

\title{
Anatomy of piriformis, obturator internus and obturator externus
}

\author{
IMPLICATIONS FOR THE POSTERIOR SURGICAL APPROACH TO \\ THE HIP
}

We dissected 20 cadaver hips in order to investigate the anatomy and excursion of the trochanteric muscles in relation to the posterior approach for total hip replacement. String models of each muscle were created and their excursion measured while the femur was moved between its anatomical position and the dislocated position. The position of the hip was determined by computer navigation.

In contrast to previous studies which showed a separate insertion of piriformis and obturator internus, our findings indicated that piriformis inserted onto the superior and anterior margins of the greater trochanter through a conjoint tendon with obturator internus, and had connections to gluteus medius posteriorly. Division of these connections allowed lateral mobilisation of gluteus medius with minimal retraction. Analysis of the excursion of these muscles revealed that positioning the thigh for preparation of the femur through this approach elongated piriformis to a maximum of $182 \%$, obturator internus to $185 \%$ and obturator externus to $220 \%$ of their resting lengths, which are above the thresholds for rupture of these muscles.

Our findings suggested that gluteus medius may be protected from overstretching by release of its connection with the conjoint tendon. In addition, failure to detach piriformis or the obturators during a posterior approach for total hip replacement could potentially produce damage to these muscles because of over-stretching, obturator externus being the most vulnerable.

- L. B. Solomon, MD, PhD, FRACS, Associate Professor Y. C. Lee, MBBS, Orthopaedic Registrar

S. A. Callary, BAppSc, Medical Scientist

D. W. Howie, PhD, FRACS Professor

Department of Orthopaedics Level 4, Bice Building, Royal Adelaide Hospital, North Terrace, Adelaide, South Australia 5000, Australia.

M. Beck, MD, PD, Head of Department

Department of Orthopaedics Lucerne Kantonsspital,

Spitalstrasse, 6004 Lucerne,

Switzerland.

Correspondence should be sent

to $\mathrm{Dr}$ L. B. Solomon; e-mail:

bogdansolomon@mac.com

(C)2010 British Editorial Society of Bone and Joint Surgery doi:10.1302/0301-620X.92B9. $23893 \$ 2.00$

$J$ Bone Joint Surg $[\mathrm{Br}]$ 2010;92-B:1317-24. Received 5 November 2009; Accepted after revision 23 April 2010

Release of soft tissue during total hip replacement (THR) should be balanced between 'sufficient' to perform the surgery safely and to allow adequate insertion of an implant and 'less invasive' to preserve soft tissue, which may lead to less post-operative pain, ${ }^{1}$ quicker rehabilitation $^{2}$ and a more stable joint. ${ }^{3}$

The posterior approach to the hip is one of the most commonly used for THR and involves detachment of the tendons of piriformis, obturator internus and obturator externus as they insert into the greater trochanter. ${ }^{4-6}$ These muscles are stabilisers of the hip and their detachment has been implicated in the higher rates of dislocation reported after THR performed through a posterior approach. ${ }^{7-11}$

In recent years, THR through minimal or less invasive surgery, which involves restricted release of tendons and muscles, has gained popularity. Most reports of less invasive posterior approaches to the hip advocate preservation of either piriformis ${ }^{2,12,13}$ or obturator externus. ${ }^{3}$ In spite of the claims of less surgical trauma and better preservation of soft tissue, objective investigations using gait analysis have been unable to show early improved function after THR performed through minimally invasive surgery compared with the use of classic techniques. ${ }^{14}$ Although this was attributed to the quick recovery of muscles released at the time of surgery during traditional approaches, ${ }^{4}$ an alternative explanation is unappreciated surgical damage related to forceful retraction or limb manipulation during minimally invasive surgery. Intra-operative electromyography has shown that muscular retraction duringTHR can partially denervate the muscles involved. ${ }^{15}$

In the absence of adequate release of soft tissues, fractures ${ }^{16}$ and avulsion and rupture of muscle can occur after excessive manipulation of the thigh. When a muscle is at its normal resting length, the individual sarcomeres have a length of approximately $2 \mu \mathrm{m} .{ }^{17,18}$ At this length, all the actin and myosin filaments overlap, producing maximum active tension for optimal contraction. As the muscle is progressively lengthened, these filaments are progressively pulled apart and active tension in the 


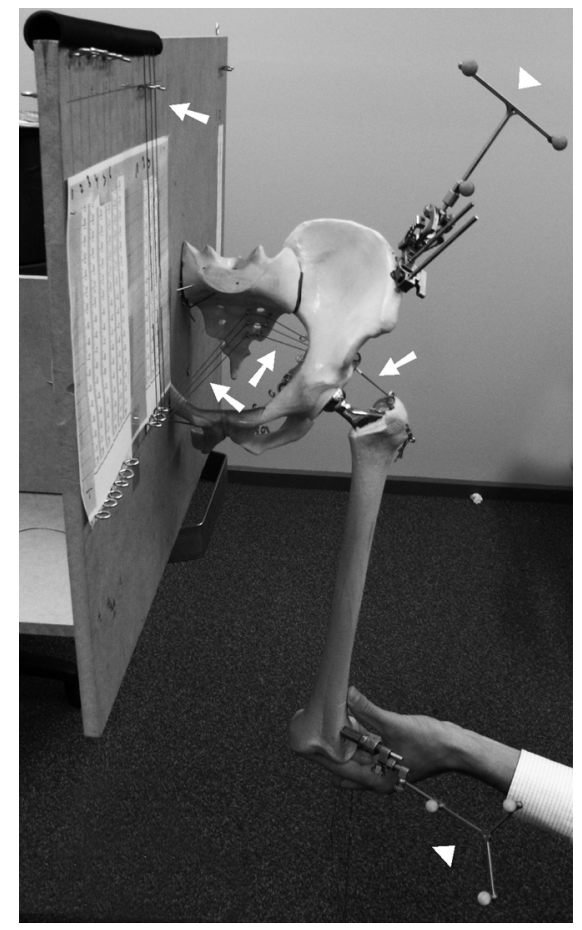

Fig. 1a

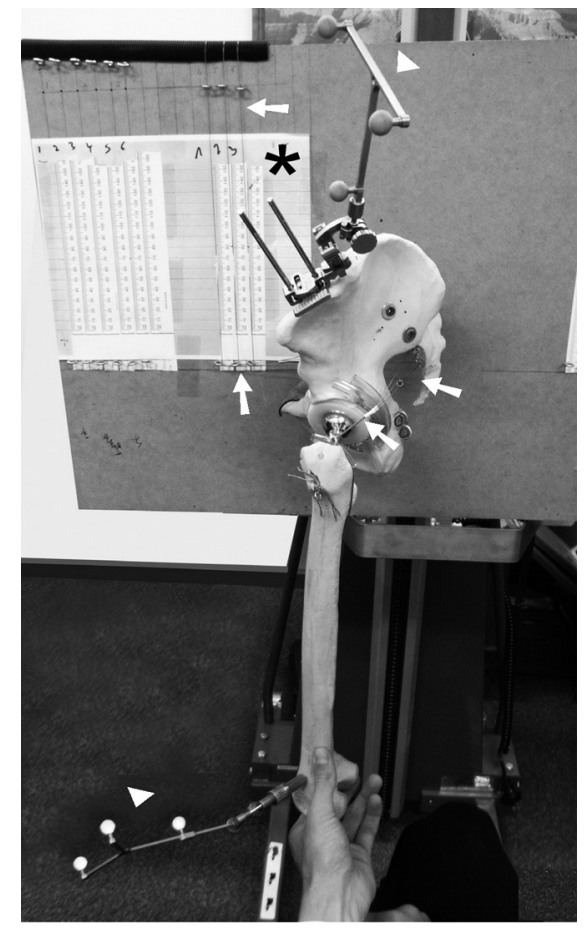

Fig. 1b

Anteroposterior a) and lateral b) photographs showing the string model of piriformis (arrows). This model was used to measure muscle excursion by computer navigation (arrowheads - pelvic and femoral sensors for computer navigation). This was measured as string movement on the scaled paper on the board to which the model was attached $(*)$. Upward movement was equivalent to muscular shortening while downward movement indicated muscular lengthening.

sarcomere decreases. When a muscle is stretched beyond approximately $180 \%$ of its normal length, or the length of the sarcomere is stretched beyond $3.6 \mu \mathrm{m},{ }^{17,18}$ all the actin and myosin filaments are pulled apart entirely, and at this length ${ }^{18}$ the muscle is damaged beyond repair.

With the exception of gluteus minimus, ${ }^{19}$ the insertion of the trochanteric muscles is poorly described and their function reported inconsistently. Traditionally, the insertion of piriformis has been described as occurring through a round tendon on to the medial side of the upper border of the greater trochanter. ${ }^{20,21}$ The insertion of obturator internus on the greater trochanter has been reported as being separate from that of piriformis, anterosuperior to the piriform fossa. ${ }^{20,21}$

Detailed knowledge of the anatomy of piriformis, obturator internus and obturator externus may help in the understanding of the effects which their preservation or release may have on the exposure of the hip during the posterior approach for THR and may help to optimise this exposure while minimising muscular damage.

Our aim was to investigate the anatomy of piriformis and the two obturator muscles. Anatomical dissections of these muscles were undertaken and string models of each muscle constructed as previously described for gluteus minimus. ${ }^{19}$ The muscle excursions were measured during the positioning of the hip for a posterior approach, using computer navigation to determine the position of the femur relative to the pelvis.

\section{Materials and Methods}

A total of 20 hips were dissected from ten embalmed cadavers with no documented medical history and no findings on dissection suggestive of pathology of the hip.

Dissection was undertaken until only gluteus medius, gluteus minimus, piriformis, obturator internus and externus, the two gemelli and the bony, ligamentous and capsular structures of the pelvis remained. The origins, insertions and the relations of piriformis, obturator internus and obturator externus with their neighbouring structures were documented. A caliper was used to measure the lengths of these muscles and tendons.

On the left side of one pelvis, piriformis and the tendons of both obturators were detached from the greater trochanter, in one layer with the posterior capsule as performed during the posterior approach to the hip. After the arthrotomy, the femur was dislocated and positioned as required for its preparation during THR. On the right side of the same pelvis, piriformis and the tendons of the obturators were similarly detached from the greater trochanter and the connection between gluteus medius and 


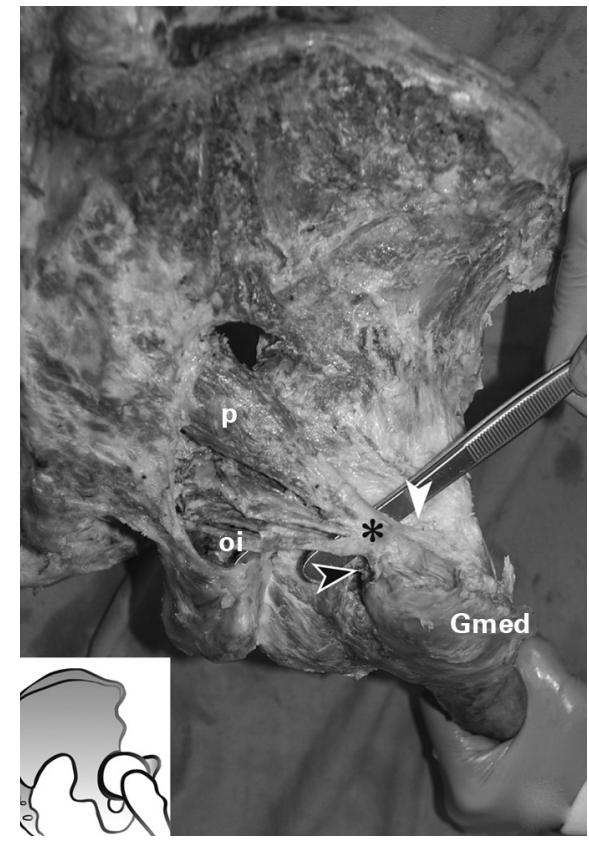

Fig. $2 a$

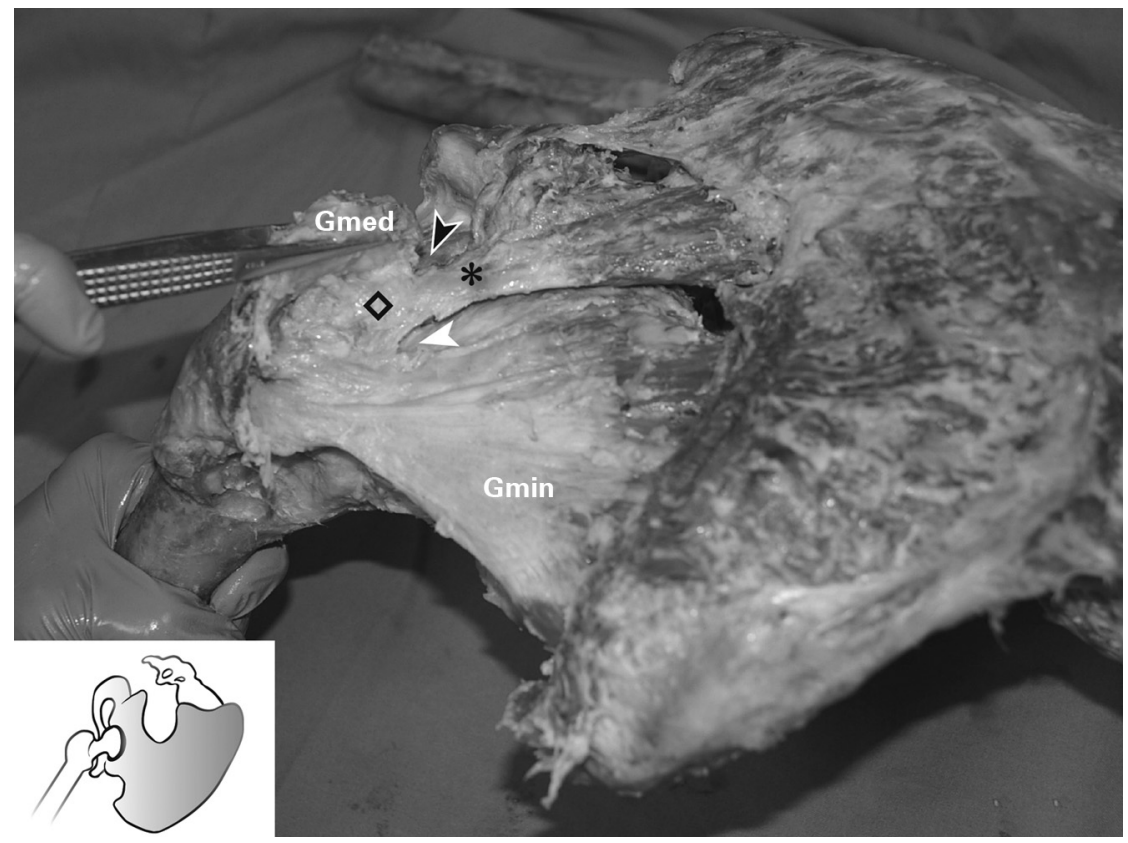

Fig. $2 b$

Superior a) and anterosuperior b) photographs of the conjoint tendon $\left(^{*}\right)$, gluteus medius (Gmed) and minimus (Gmin) insertion on the greater trochanter. The conjoint tendon is inserted over the entire superior and anterior margins of the greater trochanter (white arrowhead, capsular expansion of conjoint tendon; black arrowhead, connection between the conjoint tendon and gluteus medius; $p$, piriformis; and oi, obturator internus; 0 , space between gluteus medius and the conjoint tendon on the lateral aspect of the greater trochanter anterior to the fibrous connection between the two).

piriformis, located between the posterior margin of gluteus medius and the superior margin of the greater trochanter, was released down to the superior border of the greater trochanter. After the arthrotomy, the femur was dislocated and positioned as required for THR. The degree of retraction of gluteus medius necessary to expose the medial aspect of the trochanter was then compared between the two sides.

Plastic pelvis and femur sawbones (Models 4060 and 2167; Synbone AG, Malans, Switzerland) in combination with THR prostheses, were used to create string models for each of piriformis, obturator internus and obturator externus based on the model of gluteus minimus previously described $^{19}$ (Fig. 1). In order to investigate the differences in function and excursion between the different parts of each muscle, it was divided into several sectors, each representing a part of the muscle with a similar orientation of its fibres as previously described. ${ }^{19}$ Piriformis was divided into three, obturator internus into six and obturator externus into five equal sectors. For each muscle the most inferior sector was defined as sector 1 with the other sectors following in sequential order. Each sector was represented by a non-stretchable Cajun line $(0.431 \mathrm{~mm}$ in diameter, $36.3 \mathrm{~kg}$ resistance, Shakespeare Co, Columbia, South Carolina) firmly fixed at its insertion on the proximal femur and directed by eyelets through their origins, onto a wooden board where its excursions could be measured (Fig. 1). The excursion of each muscle sector was recorded after the femur was moved from the anatomical position to that simulating the position required for femoral preparation. The position of the femur was determined using computer navigation (VectorVision Compact Navigation Station and VectorVision hip version 3.1.0 Build 242 software; BrainLAB AG, Feldkirchen, Germany). All the measurements were repeated by three investigators (YCL, SAC, LBS) and the mean value was calculated (Table I). The position of the femur required for its preparation during a posterior approach was determined before the study by averaging the intra-operative position measured in ten THRs performed through this approach. It was found to be a combination of $60^{\circ}$ of flexion, $20^{\circ}$ of adduction, $90^{\circ}$ of internal rotation and $3 \mathrm{~cm}$ of lateral translation from the anatomical position.

When performing a THR through a posterior approach most surgeons aim to position the femoral stem in $15^{\circ}$ of anteversion. In order to achieve this, the surgeon either internally rotates the femur $90^{\circ}$ and introduces the stem at $15^{\circ}$ to the horizontal plane or internally rotates the femur $105^{\circ}$ and introduces it parallel with the horizontal plane. During this procedure, the external rotators of the hip, piriformis and the obturators, are stretched progressively with the degree of internal rotation applied to the hip. For the purposes of our study, the least amount of internal rotation was applied to the femur when simulating the position for its preparation. 


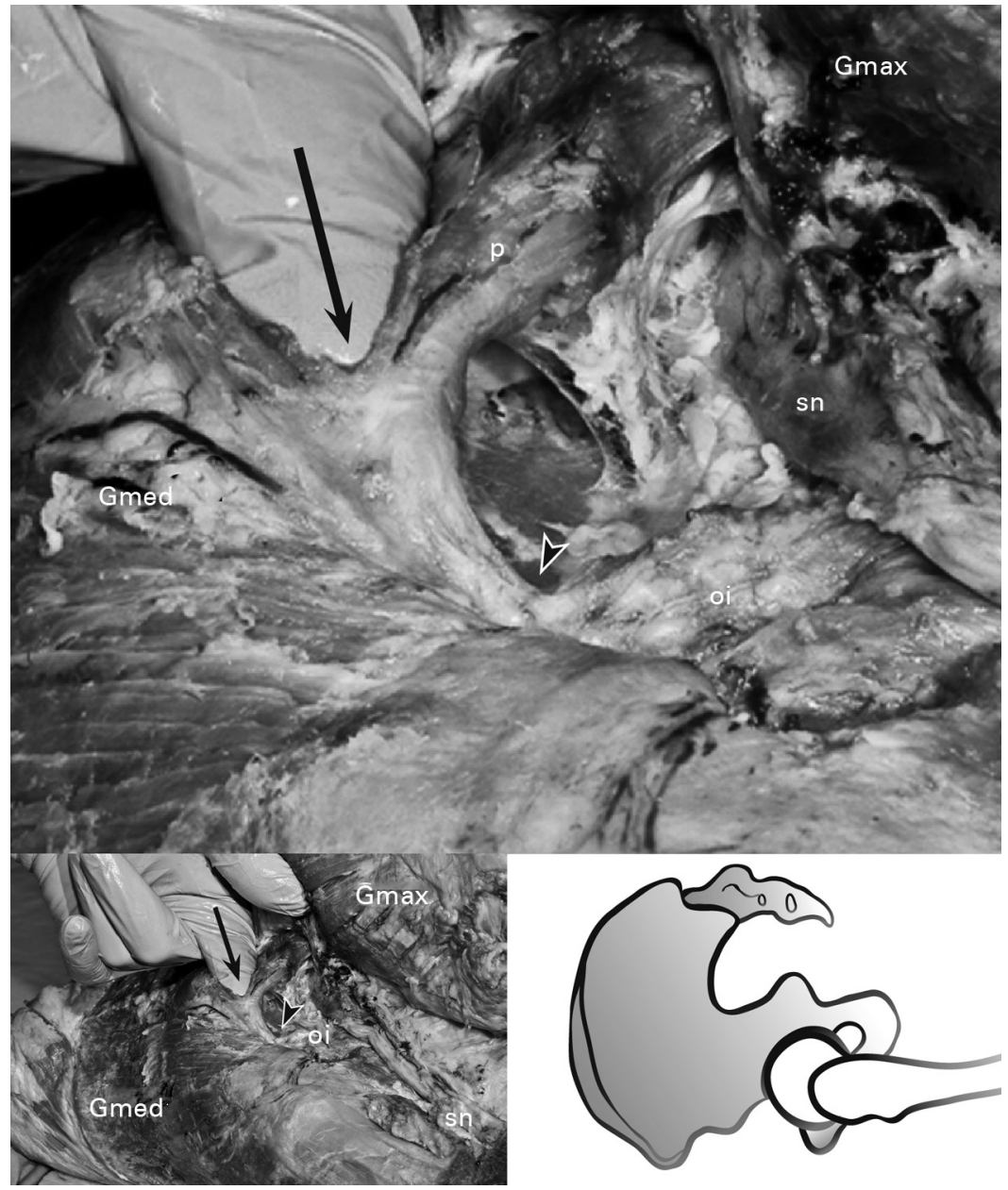

Fig. 3

Lateral photograph and diagram of a left hemipelvis. The insertions of gluteus maximus (Gmax) from the iliac crest and femur have been released and the muscle is reflected posteriorly on its sacral insertion. Gluteus medius (Gmed), piriformis $(p)$ and obturator internus (oi) are shown approaching the greater trochanter. The dissector's index finger elevates and exposes the large connection between gluteus medius and piriformis (black arrow) before piriformis joins obturator internus to form the conjoint tendon (black arrowhead) (sn, sciatic nerve).

\section{Results}

Findings of dissection. In all dissections, piriformis originated from the anterior surface of the ipsilateral half of the second to fourth sacral vertebrae. It descended laterally to leave the pelvis through the greater sciatic notch. Obturator internus had two main areas of origin, one from the rami surrounding the obturator foramen and the other from the quadrilateral plate. From its origins, the muscle fibres were directed in an almost transverse plane to leave the pelvis through the lesser sciatic notch. The cylindrically-shaped tendons of piriformis and obturator internus joined to form a conjoint tendon before their insertion on the proximal femur. The length of the conjoint tendon, defined as the distance between the point at which the two tendons united to the junction between the posterior and superior margins of the greater trochanter, varied between $0.5 \mathrm{~cm}$ and $2 \mathrm{~cm}$ (Fig. 2). Within the conjoint tendon, fibres continuing the tendon of piriformis were located mainly superiorly and then anteriorly to those continuing as the tendon of obturator internus, but the two tendons could not be completely separated after they had joined. After its formation, the conjoint tendon flattened and inserted on the superior margin and the entire medial surface of the greater trochanter as far as the proximal part of the anterior intertrochanteric line (Fig. 2). The conjoint tendon had several connections: medially with the joint capsule, laterally with the posterior margin of gluteus medius and inferiorly with the tendon of obturator externus (Figs 2 and 3). In all 20 hips, the connections of the conjoint tendon with gluteus medius and the capsule were in the sagittal plane and measured more than $1 \mathrm{~cm}$ in height. Anterior to their connection and their most posterior trochanteric insertion, the conjoint tendon and gluteus medius were separated by a fat pad, with gluteus medius taking a lateral insertion on the greater trochanter 


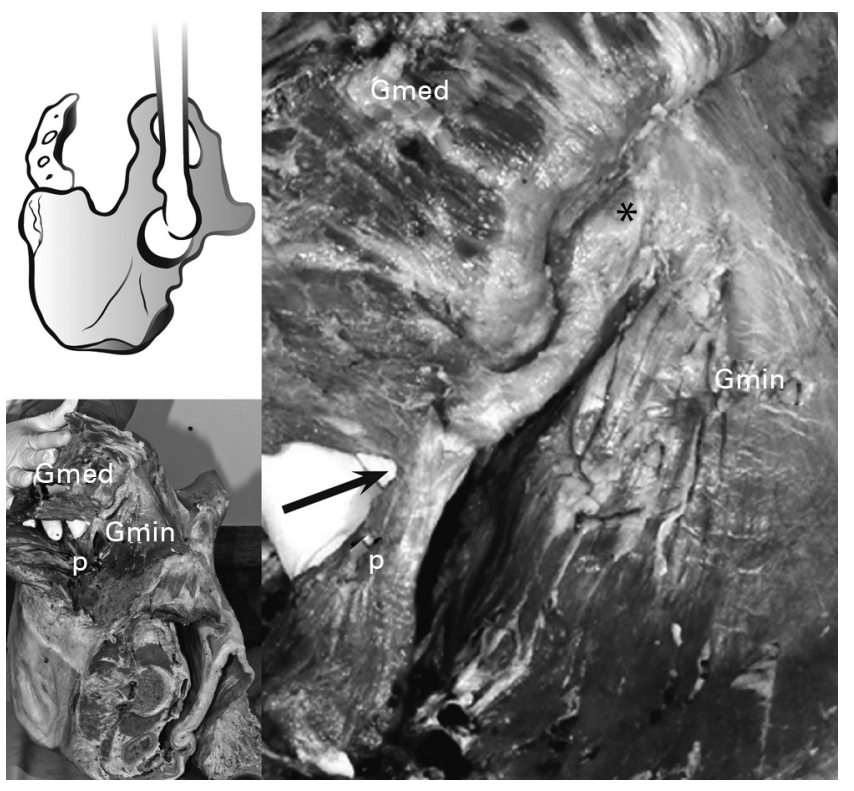

Fig. 4a

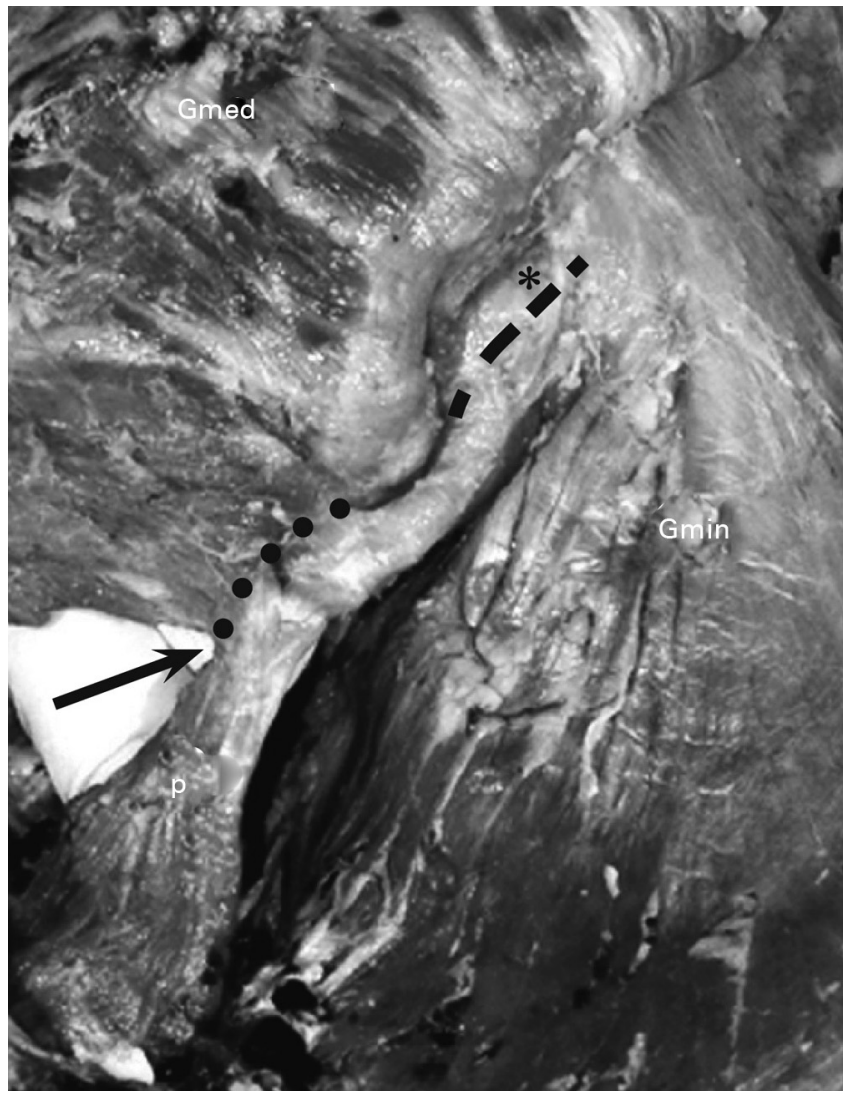

Fig. $4 b$

Figure 4a-Superior photograph and diagram of the insertions of left gluteus medius (Gmed), piriformis (p) and gluteus minimis (Gmin) on the greater trochanter, and the fat pad $\left({ }^{*}\right)$ which separates the trochanteric insertion of gluteus medius and piriformis. The dissector's right hand elevates the connection between gluteus medius and piriformis (black arrow). Figure $4 \mathrm{~b}$ - Photograph of the same specimen with the dotted line illustrating the connection between gluteus medius and piriformis (black arrow). The release of this connection along the marked line intra-operatively allows the exposure of the fat pad $(*)$ which separates the trochanteric insertion of gluteus medius and piriformis and effortless retraction, thereby protecting gluteus medius during THR performed through a posterior approach. The interrupted line marks the superior margin of the greater trochanter.

(Figs $2 \mathrm{~b}, 4$ and 5). Apart from a fibrous connection with the joint capsule, the conjoint tendon was easily separated medially and inferiorly from the femoral neck and the capsule (Fig. 2).

Complete release of the connection between the conjoint tendon and gluteus medius allowed the superior margin of the greater trochanter to be uncovered with ease from the deep surface of gluteus medius and thus the entire medial surface of the greater trochanter could be exposed without the need to retract gluteus medius. By contrast, with incomplete release of the connection between the conjoint tendon and gluteus medius, the latter remained tethered to the medial surface and superior margin of the greater trochanter and retraction of the posterior margin of gluteus medius was needed to expose most of the medial surface of the greater trochanter.

In all dissections, obturator externus originated from the external bony margin of the obturator foramen and was directed laterally, passing like a sling under the femoral neck to insert into the piriformis fossa through a cylindrical tendon. The tendon of obturator externus had connections with the joint capsule and the conjoint tendon in 16 of 20 specimens. When present, these connections were small fibrous bridges with a height of $1 \mathrm{~mm}$ to $2 \mathrm{~mm}$.

Piriformis had a mean total length of $14 \mathrm{~cm}$, its tendon measured a mean of $10 \mathrm{~cm}$ and the length of its muscle fibres ranged between $5 \mathrm{~cm}$ and $10 \mathrm{~cm}$ in every specimen. Obturator internus had a mean total length of $16 \mathrm{~cm}$, its tendon measured a mean of $10 \mathrm{~cm}$ and the length of its muscle fibres ranged from $4.0 \mathrm{~cm}$ to $7.5 \mathrm{~cm}$ in every specimen. Obturator externus had a mean total length of $11 \mathrm{~cm}$, its tendon measured a mean of $5 \mathrm{~cm}$ and the length of its muscle fibres ranged between $4.5 \mathrm{~cm}$ and $10 \mathrm{~cm}$ in every specimen. There was no difference in the length of the muscle fibres observed between each muscle sector investigated in the string models. String model and computer navigations findings. Positioning the femur to simulate the position required for femoral preparation lengthened piriformis by a mean of $3.5 \mathrm{~cm}$, obturator internus by a mean of $3.0 \mathrm{~cm}$ and obturator externus by a mean of $5.2 \mathrm{~cm}$ (Tables I and II and Fig. 6). This elongation translated to a relative muscle lengthening in each sector of up to $182 \%$ (131 to 182) for piriformis, up 


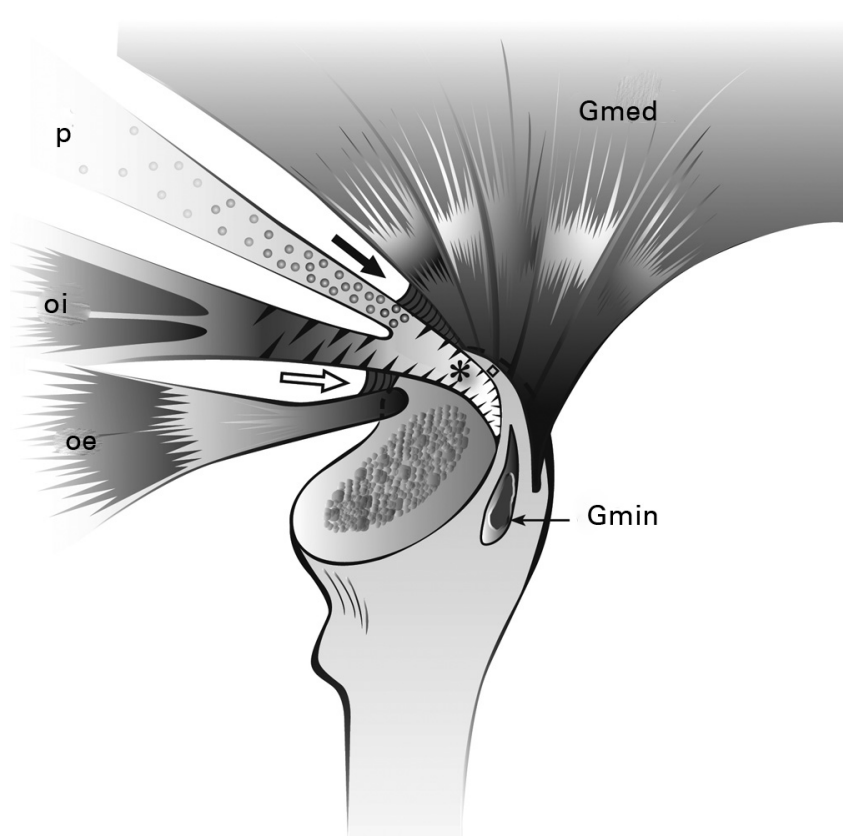

Fig. 5

Diagram showng the proximal left femur after removal of the femoral head illustrating the muscular insertion on the greater trochanter (Gmed, gluteus medius; Gmin, gluteus minimus; p, piriformis; oi, obturator internus; ${ }^{*}$, conjoint tendon; oe, obturator externus; black arrow, connection between the conjoint tendon and gluteus medius; $\diamond$, space separating the conjoint tendon and insertion of the tendon of gluteus medius on the lateral side of the greater trochanter, anterior to their connection; and white arrow, fibrous connection between conjoint tendon and obturator externus).

to $185 \%$ (143 to 185$)$ for obturator internus, and up to $220 \%$ (150 to 220 ) for obturator externus.

\section{Discussion}

A detailed knowledge of the anatomy of piriformis, obturator internus and obturator externus may help to understand the effects of the preservation or release of these tendons and their connections when exposing the hip through this surgical approach.

In all our specimens the tendons of piriformis and obturator internus converged into a conjoint tendon before inserting on the greater trochanter. The insertion of the conjoint tendon and its connection with gluteus medius has practical implications for surgery performed through this approach. If the approach involves detachment of piriformis, the posterior margin of gluteus medius remains connected to the insertion of the stump of piriformis to the superior margin of the greater trochanter, irrespective of how close the conjoint tendon is released from the trochanter. In order to allow gluteus medius to glide over the lateral aspect of the trochanter with minimal or no retraction, for the femoral preparation part of a THR, the expansion between the conjoint tendon and gluteus medius has to be released (Figs 4 and 5). In doing so the surgeon exposes
Table I. Details of the mean muscle excursion $(\mathrm{mm})$ for each sector from the anatomical position to positioning for the posterior surgical approach to the hip

\begin{tabular}{lllllll}
\hline \multirow{2}{*}{ Muscles } & \multicolumn{7}{l}{ Sector } & & & & & \\
\cline { 2 - 7 } & $\mathbf{1}$ & $\mathbf{2}$ & $\mathbf{3}$ & $\mathbf{4}$ & $\mathbf{5}$ & $\mathbf{6}$ \\
\hline Piriformis & 37.6 & 35.6 & 33.0 & & & \\
Obturator externus & 53.0 & 52.6 & 52.3 & 51.6 & 51.6 & \\
Obturator internus & 28.0 & 28.6 & $\mathbf{2 8 . 6}$ & 29.3 & 30.0 & 32.6 \\
\hline
\end{tabular}

Table II. Details of the mean (range, $\mathrm{mm}$ ) muscle excursion from the anatomical position to that for the femoral preparation required for a posterior surgical approach to the hip

\begin{tabular}{ll}
\hline Muscles excursion & Mean (range) \\
\hline Piriformis & 35.4 (31.0 to 41.0$)$ \\
Obturator externus & 52.2 (50.0 to 54.0) \\
Obturator internus & 29.5 (26.0 to 34.0$)$ \\
\hline
\end{tabular}

the fat pad which separates the insertion of gluteus medius to the lateral aspect of the greater trochanter and the insertion of the conjoint tendon to the superior margin of the trochanter (Figs 2b, 4 and 5).

If piriformis is preserved, the conjoint tendon should be divided between piriformis and obturator internus as close as possible to the anterior aspect of the greater trochanter. The tendon of piriformis can then be released from its footprint up to the superior margin of the greater trochanter until the fat pad between piriformis and gluteus medius is exposed. Release of the piriformis component from the conjoint tendon, with preservation of its connection to gluteus medius, will maintain its continuity to the greater trochanter. This also reduces the tension on piriformis while the femur is positioned for its preparation during THR. Exposing the fat pad between piriformis and gluteus medius allows the two tendons to glide over the lateral aspect of the trochanter, during femoral preparation with minimal or no retraction. The same space between the trochanteric insertions of piriformis and gluteus medius is exposed during the trochanteric slide osteotomy for surgical dislocation of the hip. In this technique, the space between piriformis and gluteus medius is opened by sacrificing the most posterior fibres of gluteus medius, which remain attached to piriformis, and then the trochanteric slide osteotomy separates gluteus medius, which is inserted on the osteotomised part of the greater trochanter, from piriformis, inserted on the non-osteotomised part. ${ }^{22}$

By applying the knowledge gained from our dissections to our clinical practice, we have found that exposing the fat pad between gluteus medius and the greater trochanter has been the single most important surgical step in protecting this muscle during THR through a posterior approach. After detachment of piriformis and the two obturators from the greater trochanter in one layer with the hip 


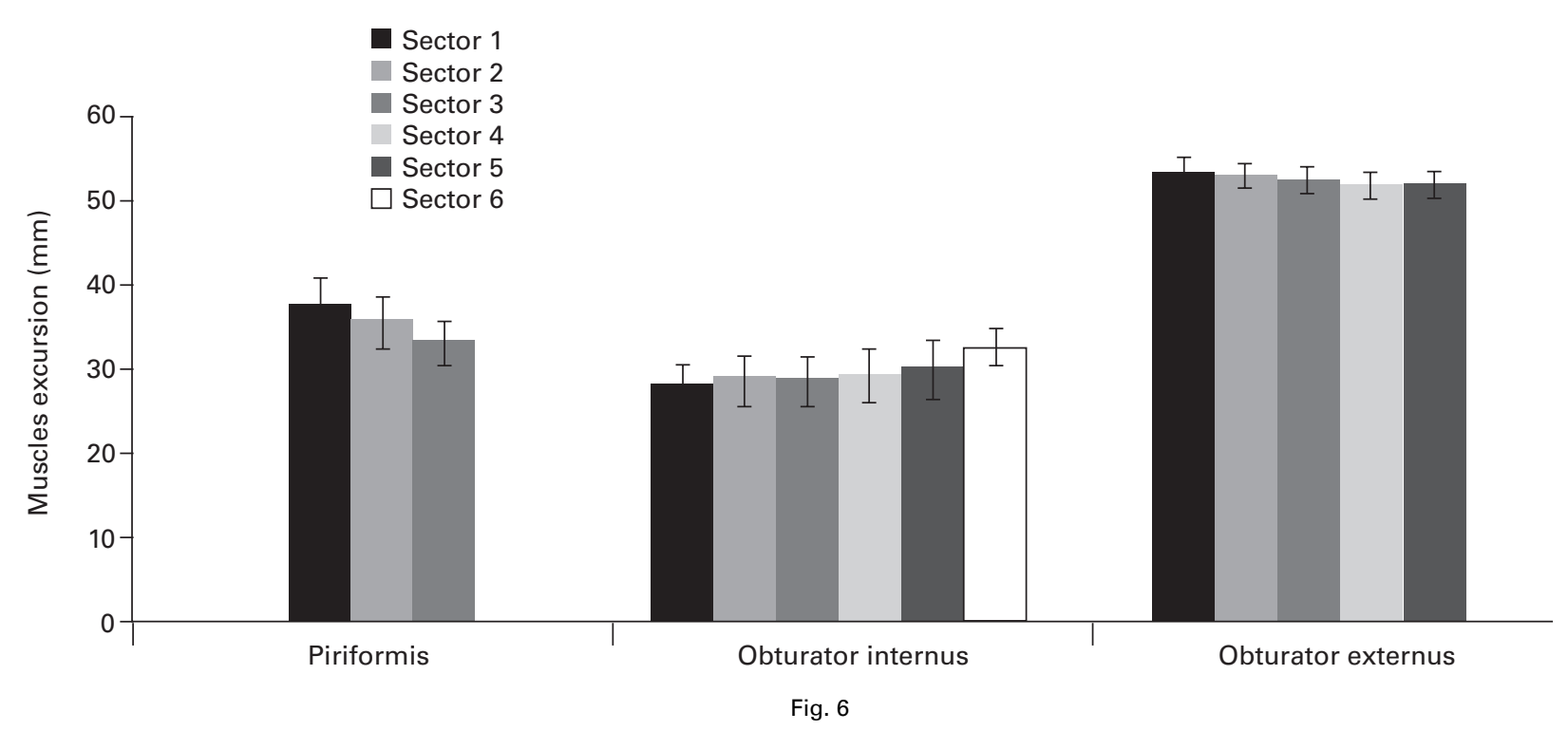

Histogram showing the excursions (mean, range) of the different sectors of piriformis, obturator externus and obturator internus while the femur is manipulated to simulate the position for its preparation during total hip replacement performed through a posterior approach.

capsule, we identify the connection between the stump of the conjoint tendon and gluteus medius. This connection is found behind the posterior margin of gluteus medius, between the tendon of gluteus medius and the insertion of the stump of the conjoint tendon on the superior margin of the greater trochanter. It is then divided, thus exposing the fat pad between gluteus medius and the greater trochanter (Fig. 4).

The tendons of obturator externus had expansions to the joint capsule and the conjoint tendon, which in most cases were very small. Such small expansions have implications for THR if this is performed through a posterior approach and the tendon of obturator externus is detached in one flap with quadratus femoris without the tendon being exposed. The blind passing of stay sutures through quadratus femoris is likely to miss going through the tendon of obturator externus. If such an approach is used and the expansions of the tendon of obturator externus are small, they will be at a high risk of being severed at the time of release without the surgeon being aware of it. A fully detached tendon of obturator externus which has not been tagged and has no capsular attachments will retract and will not be repairable at the end of the operation.

In our string models we found that the manipulation of the femur stretched the muscles investigated by $3 \mathrm{~cm}$ to $5 \mathrm{~cm}$. As tendon tissue has a very limited capacity to stretch, ${ }^{23}$ these elongations represent clinically important stretching of fibres in the muscles investigated. By showing that the intra-operative positioning of the femur can stretch piriformis and the obturators beyond their threshold for rupture, we demonstrated that failure to detach them during the posterior approach to the hip may produce partial or complete irreversible damage of these muscles. If the technique to position the femoral stem in $15^{\circ}$ of anteversion involves $105^{\circ}$ of internal rotation, rather than $90^{\circ}$, this will stretch the fibres of the muscles in question even more and further increases the risk of irreversible damage to unreleased muscles. A similar effect may be seen if an increased amount of lateral translation is applied to the femur, as may be required in large and/or short patients. As an alternative to detaching piriformis, division of the conjoint tendon and release of piriformis from the greater trochanter with preservation of its connection to gluteus medius relaxes the muscle while maintaining its continuity with the greater trochanter through gluteus medius. The effect of such a release of piriformis on the excursion of the muscle while the femur is moved from the anatomical position to that which simulates the position required for femoral preparation could not be performed in this study, but theoretically such a step would reduce the tension on piriformis during the operation.

One of the proposed causes for the higher dislocation rates of THR performed through a posterior approach is the detachment of the posterior capsule and short external rotators. ${ }^{6}$ Numerous authors have reported a reduced rate of dislocation when the posterior capsule and short external rotators have been repaired. ${ }^{6,7,24-27}$ However, despite the repair of the tendons of the obturators and piriformis, the rates of dislocation after the posterior approach for THR are still higher than when this operation is performed through other approaches. ${ }^{28}$ In order to reduce further the rate of dislocation after the posterior approach, less invasive surgery with preservation of piriformis or obturator externus was introduced giving better results when compared with those of the standard posterior approach., ${ }^{2,3}$

Our findings should caution surgeons against indiscriminate preservation of piriformis or the obturator muscles 
while performing a less invasive posterior approach for THR. Our study cannot help to identify pre-operatively the patient at risk or quantify the amount of damage caused by the manipulation of the thigh during femoral preparation through a posterior approach to the hip as a result of an unreleased piriformis, obturator internus or obturator externus. It does, however, demonstrate that muscular damage can occur if these muscles are not detached during the posterior approach for THR.

\section{Supplementary material}

ё A further opinion by $\mathrm{Mr} \mathrm{D}$. Beverland is available with the online version of this article on our website at www.jbjs.org.uk

We thank Mr N. Talbot, Dr J. Harris and Zimmer Pty Ltd., Frenchs Forest, New South Wales, Australia for providing the computer navigation system used in our study and for their technical support in operating this system. We also thank Dr K. Stiller for her help in reviewing and editing the manuscript.

No benefits in any form have been received or will be received from a commercial party related directly or indirectly to the subject of this article.

\section{References}

1. Dorr LD, Maheshwari AV, Long WT, Wan Z, Sirianni LE. Early pain relief and function after posterior minimally invasive and conventional total hip arthroplasty: a prospective, randomized, blinded study. J Bone Joint Surg [Am] 2007;89-A:1153-60.

2. Khan RJ, Fick D, Khoo P, et al. Less invasive total hip arthroplasty: description of a new technique. J Arthroplasty 2006;21:1038-46.

3. Kim YS, Kwon SY, Sun DH, Han SK, Maloney WJ. Modified posterior approach to total hip arthroplasty to enhance joint stability. Clin Orthop 2008;466:294-9.

4. Gibson A. Posterior exposure of the hip joint. J Bone Joint Surg [Br] 1950;32-B:183-6.

5. Hoppenfeld S, de Boer P. Surgical exposures in orthopaedics: the anatomic approach. Third ed. Philadelphia: Lippincott Williams \& Wilkins, 2006.

6. Suh KT, Park BG, Choi YJ. A posterior approach to primary total hip arthroplasty with soft tissue repair. Clin Orthop 2004;418:162-7.

7. Kwon MS, Kuskowski M, Mulhall KJ, et al. Does surgical approach affect total hip arthroplasty dislocation rates? Clin Orthop 2006;447:34-8.

8. Ritter MA, Harty LD, Keating ME, Faris PM, Meding JB. A clinical comparison of the anterolateral and posterolateral approaches to the hip. Clin Orthop 2001;385:95-9.

9. Sierra RJ, Raposo JM, Trousdale RT, Cabanela ME. Dislocation of primary THA done through a posterolateral approach in the elderly. Clin Orthop 205;441:262-7.
10. Vicar AJ, Coleman CR. A comparison of the anterolateral, transtrochanteric, and posterior surgical approaches in primary total hip arthroplasty. Clin Orthop 1984:188:152-9.

11. Woo RY, Morrey BF. Dislocations after total hip arthroplasty. J Bone Joint Surg [Am] 1982;64-A:1295-306.

12. Prigent $\mathbf{F}$. Incidence of capsular closure and piriformis preservation on the prevention of dislocation after total hip arthroplasty through the minimal posterior approach: comparative series of 196 patients. Eur J Orthop Surg Traumatol 2008;18:333-7.

13. Procyk S. Initial results with a mini-posterior approach for total hip arthroplasty. Int Orthop 2007;31(Suppl):17-20.

14. Ward SR, Jones RE, Long WT, Thomas DJ, Dorr LD. Functional recovery of muscles after minimally invasive total hip arthroplasty. Instr Course Lect 2008;57:249-54.

15. Siebenrock KA, Rösler KM, Gonzalez E, Ganz R. Intraoperative electromyography of the superior gluteal nerve during lateral approach to the hip for arthroplasty: a prospective study of 12 patients. J Arthroplasty 2000;15:867-70.

16. Davidson D, Pike J, Garbuz D, Duncan CP, Masri BA. Intraoperative periprosthetic fractures during total hip arthroplasty: evaluation and management. $J$ Bone Joint Surg [Am] 2008;90-A:2000-12

17. Gordon AM, Huxley AF, Julian FJ. The length-tension diagram of single vertebrate striated muscle fibres. Procs Physiological Society. 1964;171:28-30.

18. Guyton C, Hall J. Textbook of medical physiology. Tenth ed. Philadelphia: WB Saunders, 2000

19. Beck M, Sledge JB, Gautier E, Dora CF, Ganz R. The anatomy and function of the gluteus minimus muscle. J Bone Joint Surg [Br] 2000;82-B:358-63.

20. Last RJ, McMinn R-MH. Last's anatomy, regional and applied. Edinburgh: Churchill Livingstone, 1990.

21. Standring S. Gray's anatomy: the anatomical basis of clinical practice. Edinburgh: Churchill Livingstone, 2005.

22. Ganz R, Gill TJ, Gautier E, et al. Surgical dislocation of the adult hip: a technique with full access to the femoral head and acetabulum without the risk of avascular necrosis. J Bone Joint Surg [Br] 2001;83-B:1119-24.

23. Kawakami Y, Kanehisa H, Fukunaga T. The relationship between passive ankle plantar flexion joint torque and gastrocnemius muscle and achilles tendon stiffness: implications for flexibility. J Orthop Sports Phys Ther 2008;38:269-76.

24. Hedley AK, Hendren DH, Mead LP. A posterior approach to the hip joint with complete posterior capsular and muscular repair. J Arthroplasty 1990;5(Suppl):57-66.

25. Pellicci PM, Bostrom M, Poss R. Posterior approach to total hip replacement using enhanced posterior soft tissue repair. Clin Orthop 1998;355:224-8.

26. Tsai SJ, Wang CT, Jiang CC. The effect of posterior capsule repair upon post-operative hip dislocation following primary total hip arthroplasty. BMC Musculoskeletal Disord 2008;9:29

27. White RE Jr, Forness TJ, Allman JK, Junick DW. Effect of posterior capsula repair on early dislocation in primary total hip replacement. Clin Orthop 2001;393:163-7.

28. Masonis JL, Bourne RB. Surgical approach, abductor function, and total hip arthroplasty dislocation. Clin Orthop 2002;405:46-53. 